\title{
Juvenile psoriatic arthritis with rash on the hands and knees: diagnostic dilemma
}

\author{
Takuma Ohnishi $^{1} \cdot$ Satoshi Sato $^{1}$ (D) $\cdot$ Zenshiro Tamaki $^{2} \cdot$ Yoji Uejima $^{1} \cdot$ Eisuke Suganuma $^{1}$ \\ Received: 19 January 2021 / Revised: 28 January 2021 / Accepted: 31 January 2021 / Published online: 4 February 2021 \\ (C) International League of Associations for Rheumatology (ILAR) 2021
}

\section{Presentation}

A 6-year-old boy was referred to our hospital with reduced range of motion of the hand for over 2 years. Otherwise, he did not have muscle weakness or myalgia. Except for a history of atopic dermatitis, he was healthy and had no family history of similar conditions. At his first visit of our hospital, he did not take any topical steroids or immunosuppressants. Physical examination revealed symmetrical swellings of metacarpophalangeal joint, proximal interphalangeal joints, and the knee joints, and their range of motion was limited without muscle weakness. Symmetrical hyperkeratotic flat red papules were noticed on the dorsum of his fingers on hands, the extensor surfaces of elbows and knees (Fig. 1a, b). Laboratory test results were within normal limits including muscle enzymes and were negative for rheumatoid factor and HLA-B27 was negative; however, antinuclear antibodies were positive, and slit lamp examination showed no signs of uveitis. Histological examination of a biopsy of the skin lesion on the hand was consistent with that of psoriasis (Fig. 1c, d). Ultrasound confirmed intra-articular effusion of the knee joint. Contrast-

Satoshi Sato

sato.satoshi@scmc.pref.saitama.jp

1 Department of Infectious Diseases and Immunology, Division of Infectious Diseases and Immunology, Saitama Children's Medical Center, 1-2 Shintoshin, Chuou-ku, Saitama-shi, Saitama 330-8777, Japan

2 Department of Dermatology, Saitama Children's Medical Center, 1-2 Shintoshin, Chuou-ku, Saitama-shi, Saitama 330-8777, Japan enhanced magnetic resonance imaging of both knees showed contrast-enhanced lesions in the upper edge of the patella and the ligaments between the patella, roughened tibia, and synovial membrane but did not involve the muscles. Hence, he was diagnosed with juvenile psoriatic arthritis (JPsA). Treatment with methotrexate was initiated, and adalimumab was added later. His arthritis improved and is now well controlled in over 2 years of follow-up.

\section{Discussion}

JPsA is an arthritis combined with psoriasis and is believed to be a subtype of juvenile idiopathic arthritis [1]. The etiology and mechanisms linking psoriasis and arthritis are unknown. Psoriasis is occasionally difficult to distinguish from eczema and atopic dermatitis [2]. Gottron's papule also resembles psoriasis $[3,4]$. Owing to its rarity in children, pediatricians have difficulties diagnosing psoriasis [5]. Clinical diagnosis can be complicated by similarities between lesions of psoriasis, atopic dermatitis, and Gottron's papule. Skin biopsy is recommended to make a definitive diagnosis of JPsA. 
Fig. 1 a Multiple hyperkeratotic flat red papules are seen on the back of his metacarpophalangeal and interphalangeal joints, with associated swelling of these joints. b Hyperkeratotic red papules are seen on his left knee. c Thickening of the stratum corneum, irregular club-like thickening of the epidermis, and extension of dermal papillae are observed. d Incomplete keratinization is seen in the stratum corneum, along with capillary dilation, and infiltration of inflammatory cells in the dermal papilla. Perivascular infiltration of inflammatory cells is also seen in the middle layer of the dermis
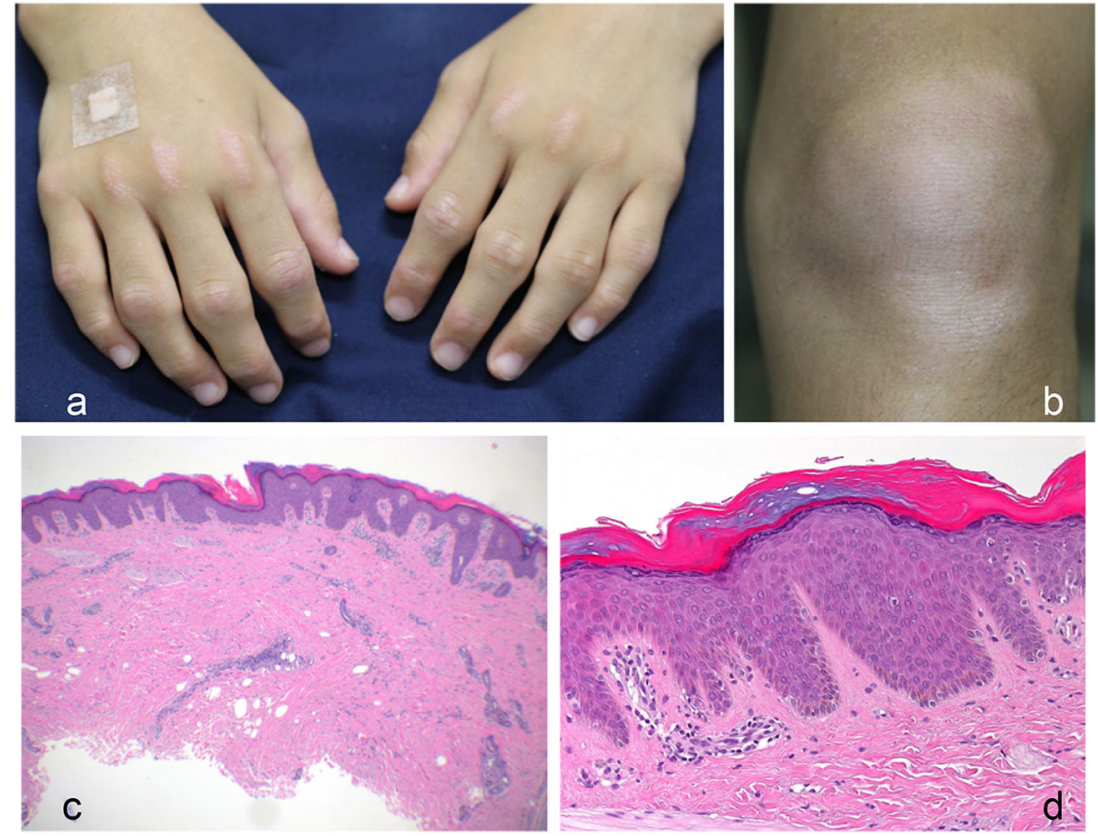

Author contribution T. O. contributed to the patient's management and drafted the manuscript. S. S. and Z. T. contributed to the patient's management and revised the manuscript. Y. U. and E. S. reviewed and revised the manuscript. All authors read and approved the final manuscript.

\section{Compliance with ethical standards}

\section{Disclosures None.}

Ethics approval The present report has been approved by Ethics Committee of Saitama Children's Medical Center.

Consent for publication Written informed consent was obtained from the patient and his guardian for publication of this case report and related clinical images.

\section{References}

1. Nigrovic PA, Sundel RP (2016) Juvenile psoriatic arthritis. In: Petty RE, Laxer RM, Lindsley CB et al (eds) Textbook of Pediatric Rheumatology, 7th edn. Elsevier, Philadelphia, pp 256-267

2. Morris A, Rogers M, Fischer G, Williams K (2001) Childhood psoriasis: a clinical review of 1262 cases. Pediatr Dermatol 18:188-198

3. Haroon M, Devlin J (2009) Gottrons's papule in amyopathic dermatomyositis mimicking psoriasis. Clin Rheumatol 28:1245-1246

4. Ricceri F, Prignano F (2013) Gottron papules: a pathognomonic sign of dermatomyositis. CMAJ 185:148

5. Michalek IM, Loring B, John SM (2017) A systematic review of worldwide epidemiology of psoriasis. J Eur Acad Dermatol Venereol 31:205-212

Publisher's note Springer Nature remains neutral with regard to jurisdictional claims in published maps and institutional affiliations. 